\title{
ON THE MEASUREMENT OF THE RATE OF INTRAMOLECULAR VIBRATIONAL ENERGY REDISTRIBUTION VIA HIGH RESOLUTION MOLECULAR BEAM OPTOTHERMAL SPECTROSCOPY
}

\author{
KEVIN K. LEHMANN, BROOKS H. PATE and G. SCOLES \\ Department of Chemistry, Princeton University, Princeton, \\ New Jersey 08544
}

\begin{abstract}
Using frequency resolved techniques we have been able to measure the rate of intramolecular vibrational energy redistribution in a number of substituted acetylenes. The method of IVR rate determination is discussed with respect to time domain measurements. The lifetimes we have determined have been quite long, ranging from a few hundred ps to a few ns. Interesting behavior of the rate as a function of the structure of the molecule has been observed. Results show sensitivity of the IVR rate to heavy mass substitution and the possibility of chromophore determined lifetimes.
\end{abstract}

KEY WORDS: High resolution infrared, IVR

\section{INTRODUCTION}

A long standing goal of physical chemistry has been the use of tunable lasers to promote or enhance bond-specific chemical reactions by exciting specific vibrational modes. Although lasers have been used to increase the rate of reactions in many instances, most of these examples have produced statistical product distributions indicating that the laser simply increases the local temperature producing an increased reaction rate, but not necessarily a reaction in a selected bond. ${ }^{1}$ It is well known that the failure of the early attempts at bond-selective chemistry is due to rapid intramolecular vibrational energy redistribution (IVR) which delocalizes the energy throughout the entire molecule on a time scale that is faster than the reaction rate. Studies which have focussed on IVR itself have shown that IVR occurs for all molecules studied once the density of background states exceeds a certain threshold (about 10-100 rovibrational states per $\mathrm{cm}^{-1}$ ). ${ }^{2}$ For even medium sized molecules ( $<10$ atoms) this onset occurs at very low energy, and, in particular, is also observed in the fundamentals of several $\mathrm{X}-\mathrm{H}$ bond stretches.

Although it is now well known that IVR occurs even at low energies, there is comparatively little quantitative information available on the time scales involved. The linewidths of overtone vibrational bands have been used as measures of the IVR rate,${ }^{3}$ but, as shown by later molecular beam measurements, ${ }^{4}$ these spectra can be 
Table 1 Density of states ${ }^{a}$ for several substituted acetylenes at the fundamental and first overtone

\begin{tabular}{lll}
\hline Molecule & $\rho(v=1)$ & $\rho(v=2)$ \\
\hline Propyne & 0.3 & 10 \\
Trifluoropropyne & 10 & 1,000 \\
Terbutylacetylene & 704 & $1 \times 10^{6}$ \\
Trimethylsilylacetylene & $2 \times 10^{4}$ & $5 \times 10^{7}$ \\
Trimethylstannylacetylene & $1 \times 10^{7}$ & $6 \times 10^{10}$ \\
Propynal & 1.5 & 49 \\
Propriolyl fluoride & 4 & 193 \\
Vinyl acetylene & 4 & 320 \\
1-butyne & 75 & 3,000 \\
\hline
\end{tabular}

\footnotetext{
a The reported density of states are the number of states per $\mathrm{cm}^{-1}$ calculated in the harmonic approximation through a direct count algorithm. The values reported in this table are not the full density of states but the density of states with the same vibrational symmetry as the acetylenic $\mathrm{C}-\mathrm{H}$ stretch mode. It is these states which can anharmonically couple to the acetylenic C-H stretch.
}

dominated by rotational and hot band contributions resulting in a greatly over estimated IVR rate. The great success of statistical unimolecular reaction rate theories, such as RRKM, have been taken as evidence of rapid energy redistribution. Chemical activation studies have determined IVR lifetimes of a few picoseconds in highly vibrationally excited molecules. ${ }^{5}$ However, often the initial state preparation is highly inhomogeneous and may be largely responsible for the observation of statistical product formation.

Recently high resolution frequency resolved techniques have been used to study the IVR process. ${ }^{6}$ These spectra provide a measurement of the IVR rate, as will be demonstrated in this paper. A major advantage of following this route is that the lifetime information obtained is homogeneous, meaning that the lifetime of a single rovibrational excited state can be determined. This is important since the dependence of the lifetime on the rotational quantum numbers can be used to determine the mechanism responsible for the redistribution (i.e. anharmonic couplings vs. rotationally mediated (Coriolis) couplings). In addition to lifetime information, the high resolution spectrum also provides precise transition frequencies and intensities. This information can be used to understand the nature of the underlying dynamics. For example, level spacing ${ }^{7}$ and intensity fluctuation ${ }^{8}$ statistics can be used to determine whether the corresponding classical dynamics of the vibrational motion are chaotic.

We present here results on a few substituted acetylenes which we have studied in our optothermal molecular beam spectrometer. We have measured the high resolution spectra of both the fundamental and first overtone of the acetylenic stretch in these molecules. In general, we find that the IVR lifetimes of these systems are relatively long, ranging from a few hundred picoseconds to a few nanoseconds. These lifetimes are sufficiently long that the energy deposited in the $\mathrm{C}-\mathrm{H}$ stretch will remain localized for times long enough that a collision will occur at reasonable pressures (about an atmosphere), presenting the possibility of bond-selective chem- 
Table 2 IVR lifetimes for some substituted acetylenes determined by high resolution infrared spectroscopy

\begin{tabular}{llc}
\hline Molecule & \multicolumn{2}{c}{ Vibrational level } \\
\hline 1-butyne & $\mathrm{v}=1$ & 200 \\
Terbutylacetylene & psec) \\
Trimethylsilylacetylene & $\mathrm{v}=1$ & 200 \\
Trimethylstannylacetylene & $\mathrm{v}=1$ & 2,000 \\
Propynal & $\mathrm{v}=1$ & 6,000 \\
Trifluoropropyne & $\mathrm{v}=2$ & 1,250 \\
Tertbutylacetylene & $\mathrm{v}=2$ & 2,000 \\
Trimethylsilylacetylene & $\mathrm{v}=2$ & 2,000 \\
Trimethylstannylacetylene & $\mathrm{v}=2$ & 4,000 \\
& $\mathrm{v}=2$ & $<5,000$ \\
\hline
\end{tabular}

istry. Bond-selective chemistry following direct overtone excitation has recently demonstrated in $\mathrm{H}_{2} \mathrm{O} .{ }^{9}$ However, in the region studied the density of states was not large enough for IVR to be a problem. The method of direct overtone absorption to produce bond-selective, bimolecular reactions may be generalizable as long as the IVR rate is sufficiently slow.

\section{EXPERIMENTAL}

The details of the optothermal molecular beam spectrometer used in these studies have been presented in previous papers. ${ }^{6 \mathrm{~g}, \mathrm{~h}}$ Briefly, a bolometer is used to detect the energy increase of a collimated molecular beam following optical excitation. The signal is proportional to the number of excited molecules in the beam, so the signal is linearly related to the laser power (up to the point where saturation occurs). This behavior allows the measurement of the first overtone region with similar sensitivity achieved in the fundamental because the laser used in the overtone provides about ten times more power. This power partly compensate for the factor of about 50 decrease in transition strength for the overtone excitation. The resolution of the spectrometer is $10 \mathrm{MHz}$ in the fundamental and $20 \mathrm{MHz}$ in the overtone and is determined by the non-orthogonal geometry of our plane-parallel multipass.

\section{THE CONNECTION BETWEEN FREQUENCY RESOLVED AND TIME RESOLVED MEASUREMENTS}

Frequency resolved experiments measure the energies of the molecular eigenstates and the intensities associated with an electric dipole transition to these states. For physical interpretations in terms of the vibrations of the molecule it is useful to start with the relationship between the molecular eigenstates and the anharmonic normal modes of the molecule, i.e. the normal modes treated to first order in the anharmonicity. The diagonal anharmonicity is important in the hydride stretches, providing transition strength for the overtones, for example, and so is included in the 
zero-order basis states. The model appropriate for our experiments is that of a single bright state (the acetylenic $\mathrm{C}-\mathrm{H}$ stretch anharmonic normal mode) that is coupled to a number of dark states (no transition strength from the ground state) through higher order couplings (anharmonic or Coriolis couplings).

The molecular eigenstates and eigenenergies are obtained by diagonalizing this system. For a system consisting of the bright state coupled to $(\mathrm{N}-1)$ dark states the diagonalization results in $\mathrm{N}$ eigenstates. An eigenstate can be written in general as

$$
\phi_{i}=\mathrm{C}_{i, \text { bright }} \Psi_{\text {bright }}+\sum_{j}^{\mathrm{N}-1} \mathrm{C}_{i, j} \Psi_{j}
$$

where the second sum is over the zero-order dark states. The transition strength from the ground state to any individual eigenstate is simply $\left|C_{i, \text { bright }}\right|^{2}$, where $C_{i \text {,bright }}$ is the coefficient of the zero-order bright state in the expansion given in Eq. 1. This result comes from the assumption that only the bright state carries transition strength from the ground state. By compteteness the total amount of bright state character is conserved and so typically as the number of coupled states is increased the amount of bright state character in any single eigenstate is decreased, i.e. the bright state is diluted.

If a single eigenstate is optically excited it will simply decay with its radiative lifetime. In general, however, a finite duration optical pulse will excite a number of eigenstates simultaneously since the eigenstates can be very closely spaced so that many will fall under the frequency bandwidth of the pulsed excitation. In the limiting case of a weak, very short optical pulse the initially prepared state is simply ${ }^{6 \mathrm{~h}}$

$$
\Psi(0)=\sum_{i} \mathrm{C}_{i, \text { bright }}^{*} \phi_{i}
$$

which is simply the anharmonic normal mode bright state.

The time evolution of this initially prepared state can be calculated using the fluorescence theroem. ${ }^{10}$ The fluorescence intensity is given by

$$
\mathrm{I}_{f l} \propto|\langle\Psi(t) \mid \Psi(0)\rangle|^{2}
$$

or using Eqs. 1 and 2

$$
\begin{aligned}
\mathrm{I}_{f l} & \propto \sum_{i}\left|\mathrm{C}_{i, \text { bright }}\right|^{4} \exp \left(-2 \gamma_{\mathrm{rad}} t\right)+ \\
& 2 \sum_{i>j}\left|\mathrm{C}_{i, \text { bright }}\right|^{2}\left|\mathrm{C}_{j, \text { bright }}\right|^{2} \cos \left[\left(E_{i}-E_{j}\right) t / h\right] \exp \left(-2 \gamma_{\mathrm{rad}} t\right)
\end{aligned}
$$

Here $\gamma_{\mathrm{rad}}$ is radiative lifetime. Since our initial state is simply the anharmonic normal mode state, the fluorescence intensity provides a measure of the time scale of energy localization in the vibration. The fluorescence intensity is the probability of finding the molecule in the anharmonic normal mode state at time $t$.

The decay contains two terms: The first term is simply the incoherent radiative decay of the individual molecular eigenstates. The second term gives the decay of the coherence created by the optical excitation. The term includes only the homogeneous set of states arising from the transition out of a single rovibrational ground 
state level. The form of Eq. (4) shows that the presence of $(\mathrm{N}-1)$ coupled states, resulting in $\mathrm{N}$ eigenstates, will result in $\mathrm{N}(\mathrm{N}-1) / 2$ beat frequencies in the fluorescence decay. As the number of coupled states increases these frequencies will tend to interfere with one another resulting in a decay with less structure. It will then become rather difficult to resolve the individual beat frequencies. Recovering a complex spectrum from a time dependent measurement becomes then quite difficult. In addition, there will be other transitions that fall under the frequency profile of the laser pulse. Transitions from different states will have their own decays and the measurement will be a sum of terms of the form of Eq. (4). This inhomogeneity will prevent the individual eigenstates from being recovered by the inversion of the time dependent measurement. Without previous knowledge of the eigenstate (frequency resolved) spectrum, the disentanglement of the time resolved spectrum is not assured.

\section{EXPERIMENTAL RESULTS}

Typically IVR is classified in three regimes: sparse, intermediate, and statistical. ${ }^{10}$ The statistical case of IVR occurs where the density of coupled states is so large that the natural (radiative) linewidth contains many states. In this case Lorentzian broadening, as expected for a true relaxation process, occurs and no individual eigenstates can be resolved in principle. If the density of states is so large that a number of states falls within the experimental resolution and the experimental linewidth is much less than the linewidth resulting from the IVR process, the result will also be the observation of a Lorentzian profile. However, in this case increased instrumental resolution would allow the resolution of the individual eigenstates. As a practical matter we will classify both of these cases as statistical case IVR. For this case the IVR lifetime $(\Gamma)$ is related to the linewidth $\delta v$ ) by

$$
\Gamma=(1 / 2 \pi \delta v)
$$

and the time resolved fluorescence experiment would find a simple exponential decay. For this case both techniques provide the same information on the IVR rate.

We have observed statistical behavior in both the fundamental and overtone spectra of $\left(\mathrm{CH}_{3}\right)_{3} \mathrm{C}-\mathrm{C} \equiv \mathrm{CH} .{ }^{6 \mathrm{~g}, \mathrm{i}}$ The fundamental and first overtone spectra of a single $\mathrm{R}$ branch transition of $\left(\mathrm{CH}_{3}\right)_{3} \mathrm{C}-\mathrm{C} \equiv \mathrm{CH}$ are shown in Figure 1. For $\left(\mathrm{CH}_{3}\right)_{3} \mathrm{C}-\mathrm{C} \equiv \mathrm{CH}$ the IVR lifetimes are about $200 \mathrm{ps}$ and $100 \mathrm{ps}$ for fundamental and overtone excitation respectively. In contrast, we find that the IVR rate in the overtone of $\left(\mathrm{CH}_{3}\right)_{3} \mathrm{Si}-\mathrm{C} \equiv \mathrm{CH}$ is twice as long as the rate in the fundamental. Furthermore, the rates found in $\left(\mathrm{CH}_{3}\right)_{3} \mathrm{Si}-\mathrm{C} \equiv \mathrm{CH}$ are dramatically slower than those found in $\left(\mathrm{CH}_{3}\right)_{3} \mathrm{C}-\mathrm{C} \equiv \mathrm{CH}$ even though the silicon substituted compound has a much larger density of states; the IVR lifetime of the fundamental is about 2 ns and the overtone lifetime is greater than about $6 \mathrm{~ns}$. This means and the lifetimes of the silicon substituted species are long enough that the vibrational energy will remain localized on time scales of mean collision times in gas phase samples.

Intermediate case behavior occurs when there are enough coupled states to drive an initial relaxation. A time resolved measurement will then exhibit an initial 

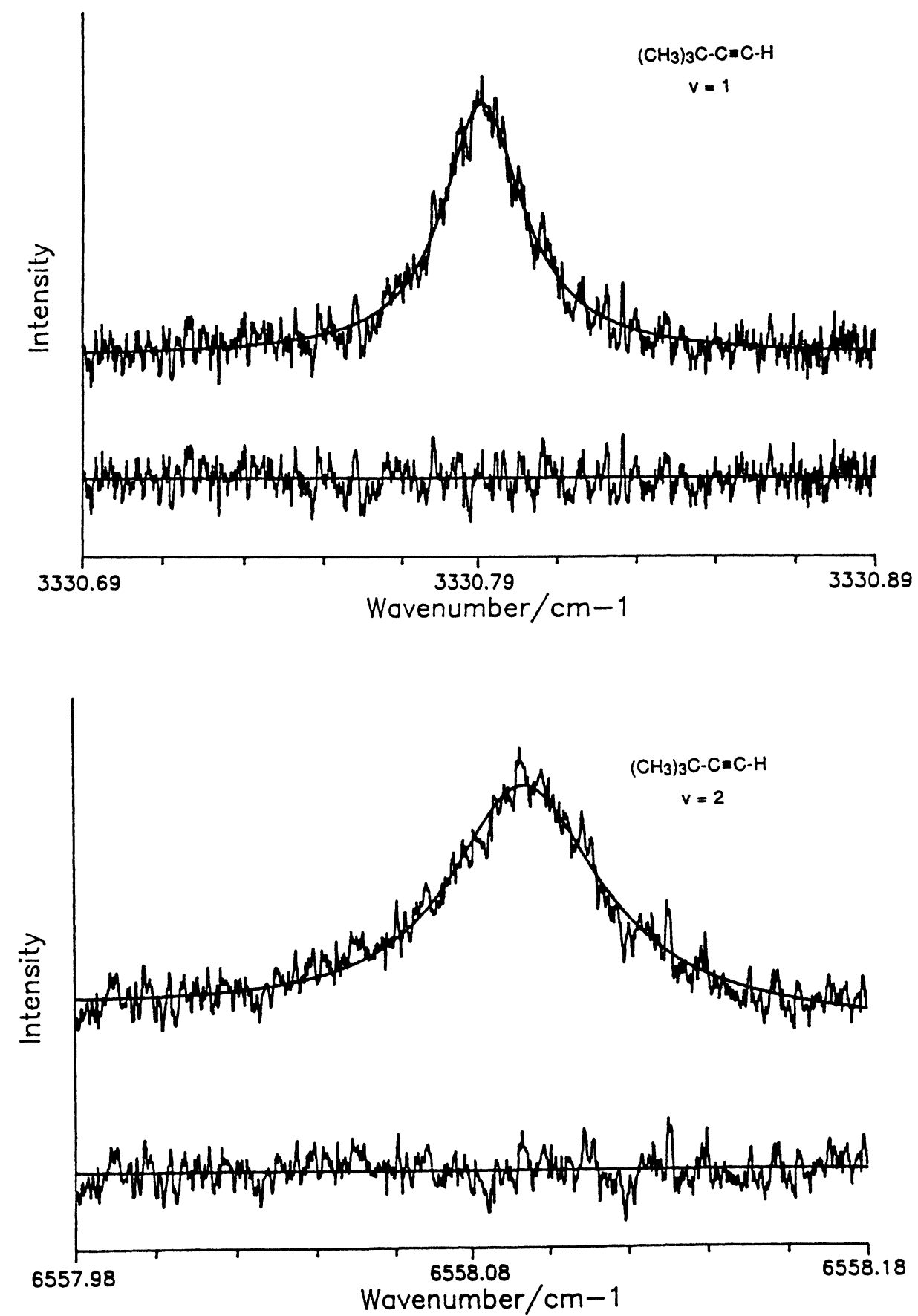

Figure 1 Statistical case spectra observed for $\left(\mathrm{CH}_{3}\right)_{3} \mathrm{C}-\mathrm{C} \equiv \mathrm{CH}$. For the fundamental (above) the $R(5)$ transition is shown. The fit to a single Lorentzian (FWHM of about $800 \mathrm{MHz}$ ) is given. The residuals of the fit are plotted below the line. The IVR lifetime is about $200 \mathrm{ps}$. For the overtone the $R(7)$ transition with its best fit Lorentzian (FWHM of about $1400 \mathrm{MHz}$ ) is shown. The lifetime is the overtone decreases to about 100 ps. Using the optothermal technique, the sensitivity in the fundamental and first overtone are comparable. 
exponential decay reflecting the overall width of the eigenstate distribution. Since there are only a finite number of levels, there will be recurrences at later times, however, these will usually have reduced amplitudes. The initial decay is then taken to measure the IVR rate. Numerical studies have shown that this behavior occurs when about ten coupled states are present. ${ }^{11}$ When fewer states are coupled, i.e. in the "sparse" IVR case, the time resolved measurement will show a few beats with large amplitude recurrences so that no initial decay of the vibrational localization can be easily identified. The frequency resolved spectrum will show a few isolated perturbations which can be studied using the traditional methods of high resolution spectroscopy. We have observed this behaviour in the fundamental of $\mathrm{CF}_{3} \mathrm{CCH},{ }^{6 f, h}$ but since this really does not represent IVR it will not be presented here.

We have observed and analyzed intermediate case behavior in the overtone of $\mathrm{CF}_{3} \mathrm{CCH} .{ }^{6 \mathrm{~h}}$ Here we present the fundamental spectrum of 1-butyne as an example of an intermediate case spectrum. This spectrum has been the subject of previous high resolution studies and the interpretation of the spectrum has been presented. ${ }^{6 a, c, e}$ Our spectrum, shown in Figure 2, shows 36 states associated with the single $1_{01}$ $\left(\mathrm{J}_{\mathrm{KaKc}}\right)$ excited rovibrational state. The time evolution of the homogeneous excited state is also shown in Figure 2. It is characterized by an initial decay of the vibrational localization, followed by a few small recurrences. The lifetime, given by the $1 / \mathrm{e}$ point of the initial decay, is about $200 \mathrm{ps}$. We note the relative smoothness of the calculated time evolution compared to the complexity of the high resolution spectrum. This is a case where retrieval of the eigenstates from the transform of the time dependent spectrum would likely be difficult. Furthermore, there are transitions in the same region which do not belong to the $1_{01}$ upper state (these are due to b-type transitions). These additional eigenstates will contribute to the time resolved spectrum, further complicating the interpretation of the data.

Lastly, we point out the similarity in the fundamental lifetimes for 1-butyne and $\left(\mathrm{CH}_{3}\right)_{3} \mathrm{C}-\mathrm{C} \equiv \mathrm{CH}$. High resolution measurements of the two conformers of 1pentyne also give IVR lifetimes on the order of $200 \mathrm{ps}^{6 \mathrm{e}}$ These results suggest that the IVR rate is chromophore dependent and does not depend on the total density of states (which varies greatly for these molecules). However, the dramatically different lifetime of $\left(\mathrm{CH}_{3}\right)_{3} \mathrm{Si}-\mathrm{C} \equiv \mathrm{CH}$ shows that the lifetime is very sensitive to molecular structure and that the dynamical nature of the acetylenic chromophore is determined by at least the first three atoms attached to the acetylenic hydrogen.

\section{CONCLUSION}

Using frequency resolved methods we have been able to determine the rate of intramolecular vibrational energy redistribution in a few substituted acetylenes. This rate information can be determined both from statistical case spectra and intermediate case spectra. Frequency resolved techniques have the advantage that truly homogeneous lifetimes can be determined. For intermediate case spectra precise frequencies and intensities are also obtained that can be analyzed to provide additional information on the vibrational dynamics. 


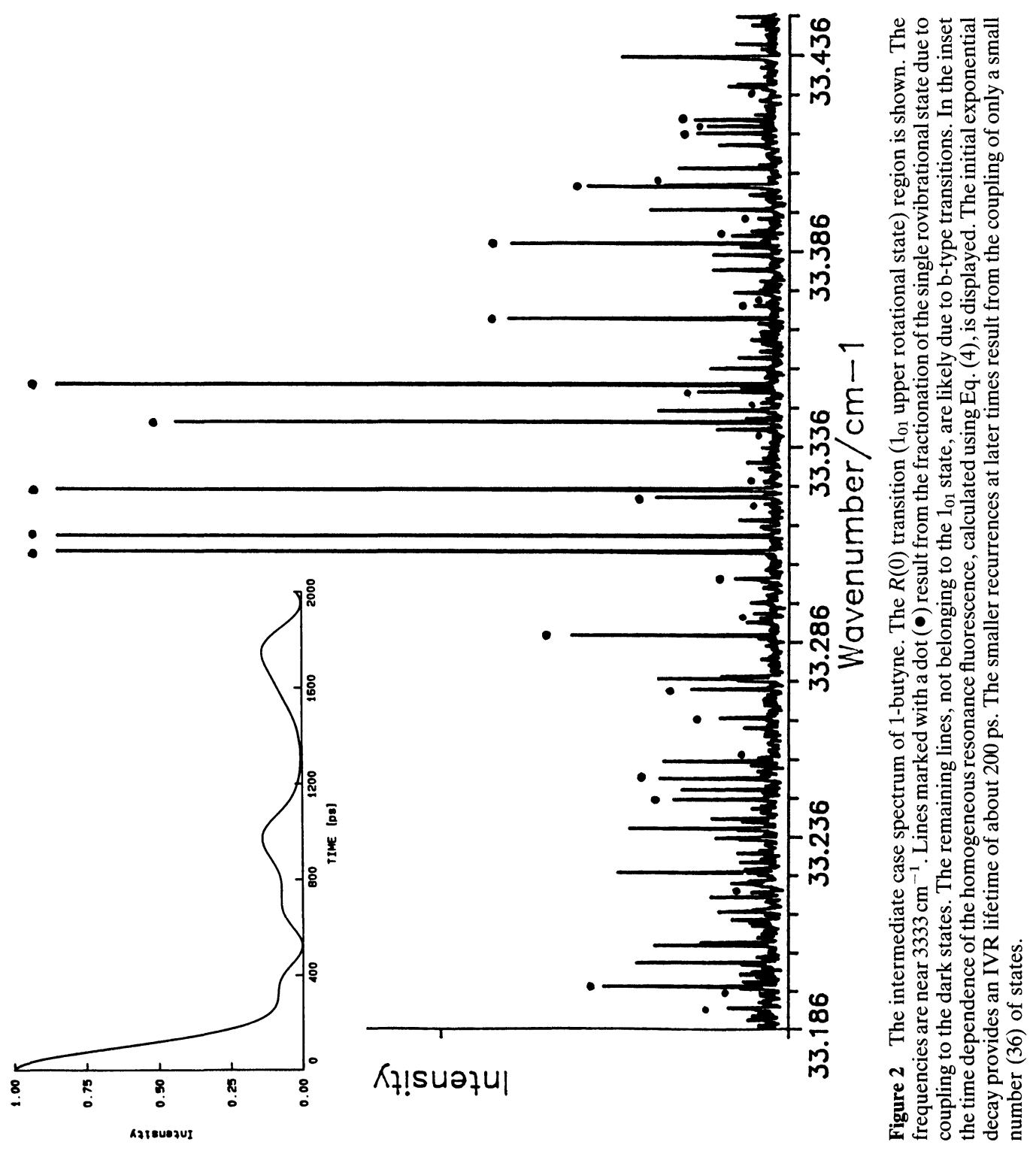


All of the lifetimes we have measured have been quite long, longer than $100 \mathrm{ps}$ and ranging up to about $10 \mathrm{~ns}$. The lifetimes of the molecules appears to be very dependent on the molecular structure, but for similar structures a chromophore dependent rate may exist. The long time scale of the vibrational redistribution suggests that direct overtone excitation of these or similar systems may result in bond specific chemical reactions. Even though a single eigenstate may contain very little contribution from the acetylenic $\mathrm{C}-\mathrm{H}$ stretch, an acetylenic $\mathrm{C}-\mathrm{H}$ stretch initial state can be created by a short optical pulse (shorter than the IVR rate). The vibrational energy will remain localized in this bond for the time set by the IVR lifetime. The length of the measured lifetimes are sufficiently long that reasonable pressure gas phase mixtures will have mean collision rates shorter than the vibrational relaxation. Bond-selective bimolecular reactions that are generalizations of the results found for $\mathrm{H}_{2} \mathrm{O}$ (where no IVR occurs) ${ }^{9}$ are then possible.

\section{Acknowledgements}

We would like to thank Erik Kerstel and Thomas Mentel who have collaborated on the IVR work in Princeton. This research has been funded by the National Science Foundation.

\section{References}

1. (a) R. L. Woodin and A. Kaldor, Adv. Chem. Phys. 47 pt. 2, 3 (1981). (b) F. F. Crim, Ann. Rev. Phys. Chem. 35, 657 (1984).

2. (a) C. S. Parmenter, Faraday Discuss. Chem. Soc., 75, 7 (1983). (b) H. L. Kim, T. J. Kulp and J. D. McDonald, J. Chem. Phys. 87, 4376 (1987).

3. K. V. Reddy, D. F. Heller and M. J. Berry, J. Chem. Phys. 76, 2814 (1982).

4. (a) R. H. Page, Y. R. Shen and Y. T. Lee, J. Chem. Phys. 88, 4621 (1988). (b) M. Scontoni, A. Boschetti, N. Oberhofer and D. Bassi, J. Chem. Phys. 93, 971 (1991).

5. I. Oref and B. S. Rabinovitch, Acc. Chem. Res. 12, 166 (1979).

6. (a) A. M. deSouza, D. Kaur and D. S. Perry, J. Chem. Phys. 88, 4569 (1988). (b) J. Go, G. A. Berthardy and D. S. Perry, J. Phys. Chem. 94, 6153 (1990). (c) G. A. Berthardy and D. S. Perry, J. Mol. Spectrosc. 144, 304 (1990). (d) A. McIlroy and D. J. Nesbitt, J. Chem. Phys. 91,104 (1989) (e) A. McIlroy and D. J. Nesbitt, J. Chem. Phys. 92, 2229 (1990). (f) K. K. Lehmann, B. H. Pate and G. Scoles, J. Chem. Soc. Faraday Trans. 86, 2071 (1990). (g) K. K. Lehmann, B. H. Pate and G. Scoles, J. Chem. Phys. 93, 2152 (1990). (h) B. H. Pate, K. K. Lehmann and G. Scoles, J. Chem. Phys., to be published. (i) E. R. Th. Kerstel, K. K. Lehmann, T. F. Mentel, B. H. Pate and G. Scoles, J. Phys. Chem., to be published.

7. T. A. Brody, J. Flores, J. B. French, P. A. Mello, A. Pandey and S. S. M. Wong, Rev. Mod. Phys. 53, 385 (1981).

8. Y. M. Engel and R. D. Levine, J. Chem. Phys. 89, 4633 (1988).

9. A. Sinha, M. C. Hsiao and F. F. Crim, J. Chem. Phys. 92, 6333 (1990).

10. K. F. Freed and A. Nitzan, J. Chem. Phys. 73, 4765 (1980).

11. F. Lahmani, A. Tramer and C. Tric, J. Chem. Phys. 60, 4431 (1974). 